[Case Report]

\title{
Vertebrobasilar Infarction Due to Bow Hunter's Syndrome in a Patient with Rheumatoid Arthritis: A Case Report
}

\author{
Yuta Yoshimatsu $^{1}$, Satoru Ide ${ }^{1 *}$, Shingo Kakeda ${ }^{2}$, Yu Murakami $^{1}$, Satoshi Fukumitsu ${ }^{1}$, Yohei Takeshita ${ }^{1}$, \\ Kenta AnaI ${ }^{1}$, Toshihiko Hamamura ${ }^{1}$, Shuhei Yoshida ${ }^{3}$, Akinori SaKaI ${ }^{3}$ and Yukunori Korogi ${ }^{1}$ \\ ${ }^{1}$ Department of Radiology, School of Medicine, University of Occupational and Environmental Health, Japan. \\ Yahatanishi-ku, Kitakyushu 807-8555, Japan \\ 2 Department of Radiology, Graduate School of Medicine, Hirosaki University, Zaifu-cho, Hirosaki 036-8563, Japan \\ ${ }^{3}$ Department of Orthopedic Surgery, School of Medicine, University of Occupational and Environmental Health, \\ Japan. Yahatanishi-ku, Kitakyushu 807-8555, Japan
}

\begin{abstract}
A 60-year-old woman with a 37-year history of rheumatoid arthritis (RA) had a sudden onset of headache. Head MRI showed acute multiple infarctions in the vertebrobasilar region, and MR angiography showed stenosis of the right vertebral artery (VA). 3D-CT angiography of the craniovertebral junction showed atlantoaxial subluxation and stenosis of the right VA just distal to the transverse foramen of $\mathrm{C} 2$, which was due to osteophytes and degenerative changes secondary to RA. Digital subtraction angiography clearly demonstrated occlusion of the right VA during rightward head rotation. Based on those findings, rotatory instability at $\mathrm{C} 1-2$ was considered as the primary cause of the vertebrobasilar infarctions, and Bow Hunter's syndrome was diagnosed. The patient underwent $\mathrm{C} 1-5$ posterior fixation, and brain infarction has not recurred.
\end{abstract}

Keywords : bow hunter's syndrome, rheumatoid arthritis, cerebral infarctions, atlantoaxial subluxation.

(Received February 28, 2021, accepted March 24, 2021)

\section{Introduction}

Bow Hunter's syndrome (BHS) is a form of vertebrobasilar insufficiency, also known as rotational vertebral artery (VA) occlusion, because of mechanical stenosis or occlusion of the VA at the subaxial or atlantoaxial joint. The common symptoms of BHS are dizziness, vertigo, syncope, dysarthria, nausea and dysphagia [1]. The flow of the VA is reduced with head rotation because of osteophytes, degenerative cervical spondylosis, or the longus colli muscle [2].

Rheumatoid arthritis (RA) often induces destruction followed by instability of the cervical spine, such as atlantoaxial subluxation (AAS). Lateral AAS is a subtype of this disorder, which usually accompanies head rotation and is observed in around $20 \%$ of cases [3]. Although they are uncommon conditions, previous investigators have reported that AAS in RA patients is related to BHS $[4,5]$. There are some reports demonstrating cerebral infarctions due to AAS in RA patients, but those were not confirmed as BHS $[3,6,7]$. To the best of our knowledge, there are only a few reported cases of cerebral infarctions induced by BHS in RA patients $[4,5]$. We herein report a case of vertebrobasilar infarction due to BHS in a patient with RA.

*Corresponding Author: Satoru IDE, Department of Radiology, School of Medicine, University of Occupational and Environmental Health, Japan. 1-1 Iseigaoka, Yahatanishi-ku, Kitakyushu 807-8555, Japan, Tel: +81-93-603-1611, Fax: +81-93-603-1612, E-mail: s-ide@med.uoeh-u.ac.jp 


\section{Case Presentation}

A 60-year-old Japanese woman presented at our hospital with sudden headache of no laterality and nausea. She had a 37-year history of RA and had been treated with Methotrexate and Tacrolimus Hydrate. Brain MRI at onset showed no hemorrhage or infarction, although the right VA was poorly visualized in MR angiography (MRA) (Figure 1). She was hospitalized with a policy of conservative treatment. She felt nausea on the second day of hospitalization and undertook MRI, which showed an acute infarction in the left cerebellar hemisphere (posterior inferior cerebellar artery: PICA territory) and in the left occipital lobe (Figure 2A and B). Although the right VA became well visualized, stenosis just distal to the $\mathrm{C} 2$ transverse foramen was demonstrated in MRA (Figure 2C).

The ischemic events were resolved and she was discharged, but she complained of vertigo one month later. MRI showed no brain infarction, but the right VA was poorly visualized again (not shown). Sagittal CT of the cervical spine demonstrated cranial settling with upward translocation of the odontoid process relative to the foramen magnum due to advanced RA (Figure 3). 3D-CT Angiography showed ankylosis through the lateral mass of the $\mathrm{C} 1$ and occipital condyle, and collapse of the lateral mass of the $\mathrm{C} 2$ and osteophytes, which resulted in stenosis of the right VA just distal to the $\mathrm{C} 2$ transverse foramen (Figure 4A-C). A coronal T1-weighted vessel wall image obtained 4 days later showed no subintimal hematoma (Figure 4D). From the stenosis and repeated poor visualization of

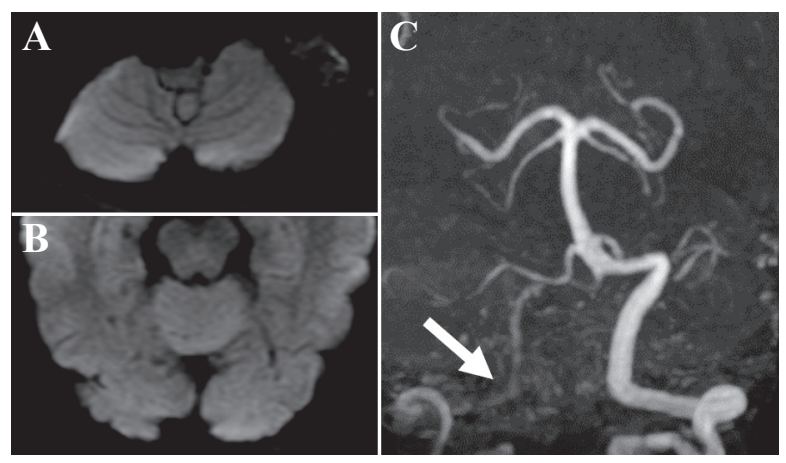

Figure 1. MRI at onset. No hemorrhage or infarction was found on MRI at this time (A and B), although the right vertebral artery is poorly visualized (arrow in $\mathrm{C}$ ). the right VA, instability at $\mathrm{C} 1-2$ was considered as the primary cause of the vertebrobasilar insufficiency, and BHS was suspected. Digital subtraction angiography (DSA) clearly demonstrated right VA occlusion with rightward neck rotation, so BHS due to rotatory AAS was diagnosed (Figure 5). The patient underwent $\mathrm{C} 1-5$ posterior fixation, and brain infarction and symptoms related to vertebrobasilar insufficiency have not recurred during 2-years of observation.

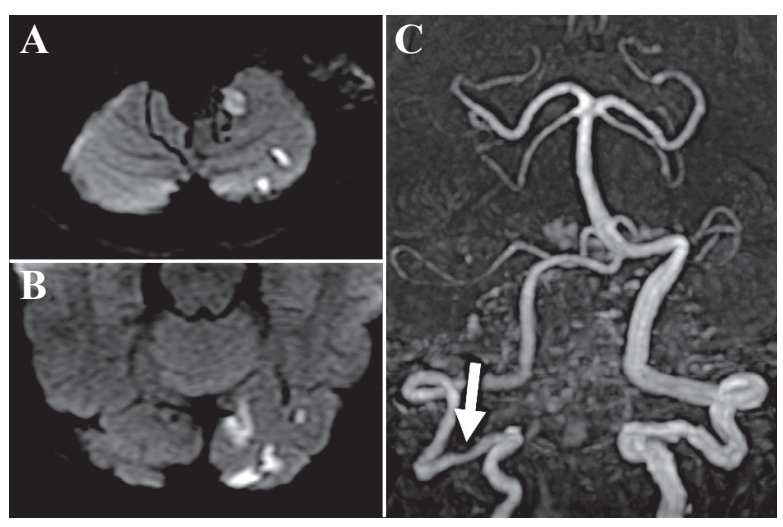

Figure 2. MRI taken on the second day of hospitalization. Diffusion-weighted image shows high-intensity lesions in the left cerebellum (A) and left occipital lobe (B), revealing multiple acute infarcts. The right vertebral artery is visualized well, and stenosis just distal to the C2 transverse foramen is demonstrated on MRA (arrow in C). Dissection flap and subintimal hematoma were not found on MRA. MRA: MR angiography.

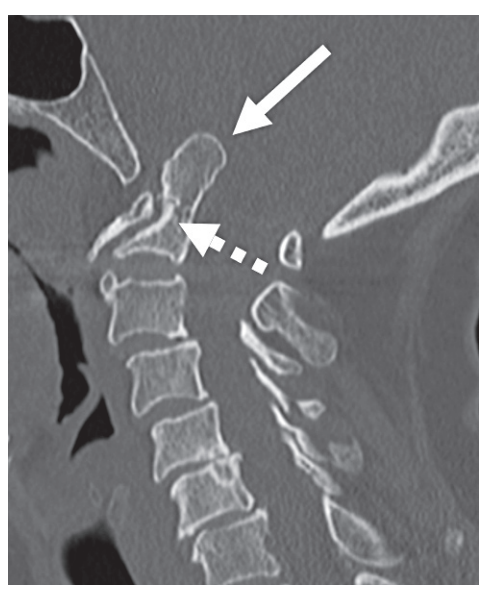

Figure 3. Sagittal CT of cervical spine. Cranial settling with upward translocation of the odontoid process relative to foramen magnum (arrow) and an anterior odontoid erosion due to advanced RA (dot arrow) are demonstrated. RA: rheumatoid arthritis 

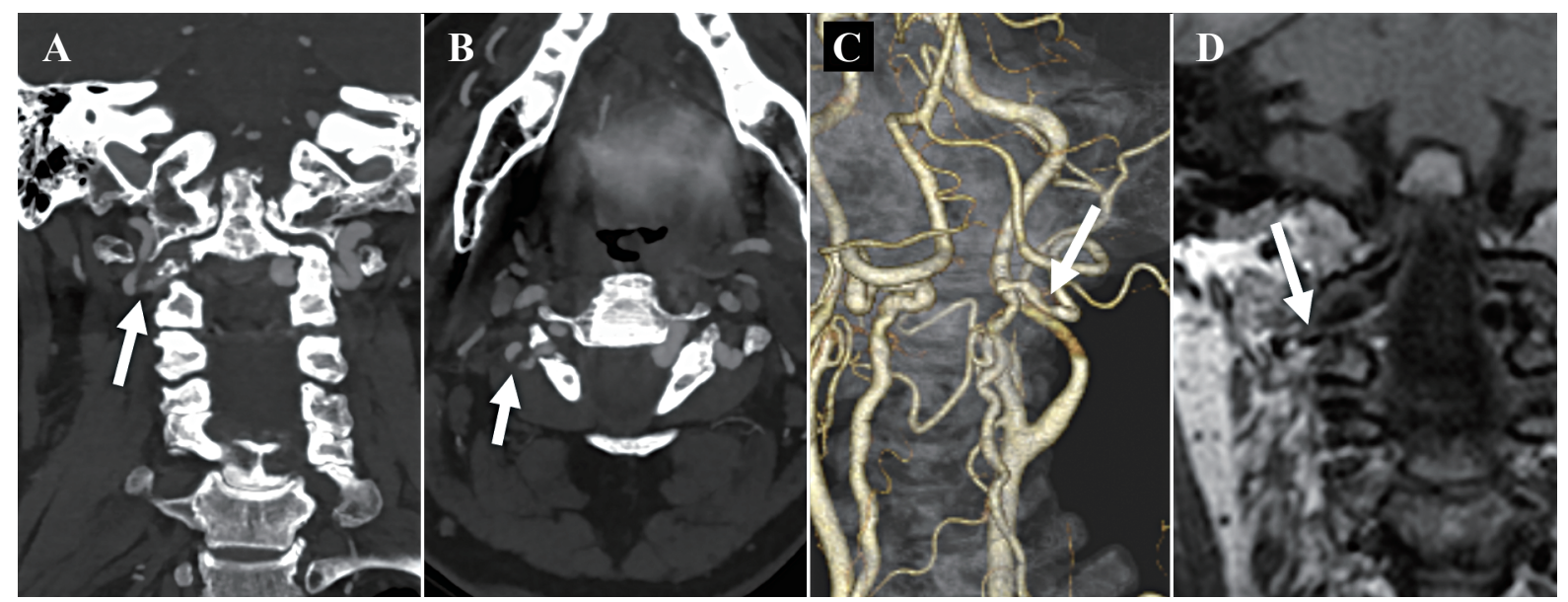

Figure 4. Angiography and T1-weighted vessel wall image of the craniovertebral junction. Ankylosis through lateral mass of $\mathrm{C} 1$ and occipital condyle, and collapse of lateral mass of $\mathrm{C} 2$ and osteophytes formation, which resulted in stenosis of the right VA just distal to the $\mathrm{C} 2$ transverse foramen, are demonstrated (arrows in A, B, and C). T1-weighted vessel wall image shows no subintimal hematoma around the narrow flow void of the right VA (arrow in D). VA: vertebral artery

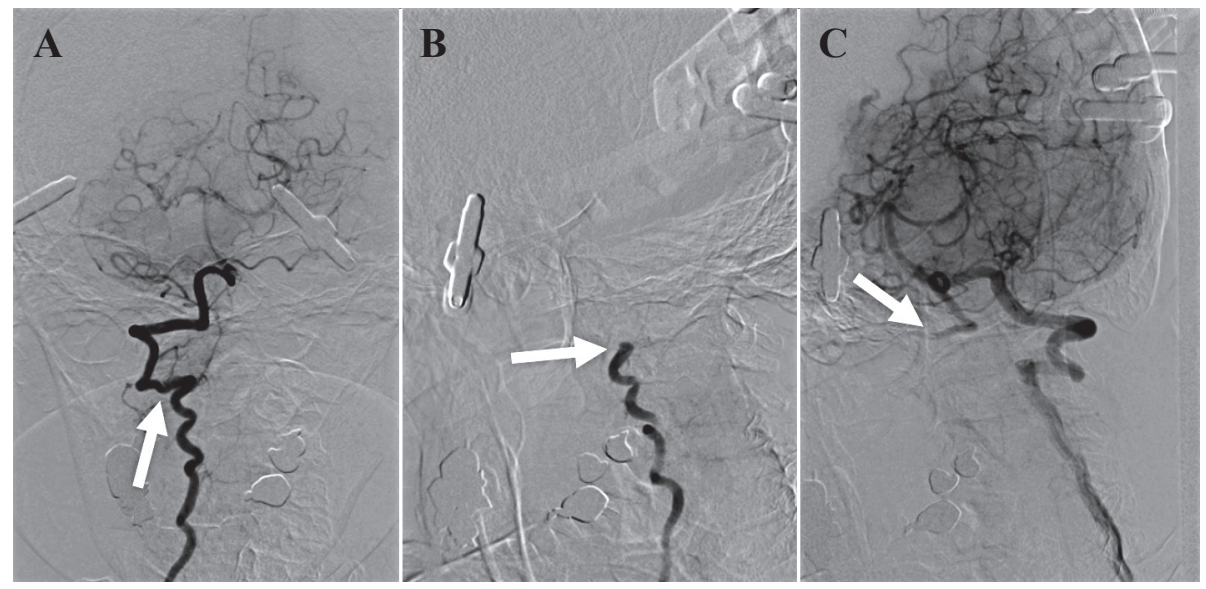

Figure 5. Digital subtraction angiography of vertebral artery (VA). The right VA shows mild stenosis just distal to the $\mathrm{C} 2$ transverse foramen in the neutral position (arrow in A). In an oblique view with the head rotated to the right side, the right VA is occluded at the C2 level (arrow in B). The left VA angiography in the same position shows retrograde flow to the right VA distal to the occlusion site through the confluence of VA (arrow in C), revealing that the right VA is occluded.

\section{Discussion}

Bow Hunter's Syndrome (BHS) is known as a disease which shows various symptoms due to cervical movement, especially lateral rotating of the neck. It is caused by ischemia because of reduction of VA flow at the time of head rotation. The underlying etiology of BHS varies. It could be intrinsic vascular disease, such as atherosclerosis or dissection, or it could be mechanical external compression of the VA during head rotation from muscular or tendinous insertions, osteophytes, or degenerative changes secondary to cervical spondylosis. According to recent retrospective reviews of BHS, osteophytes are the most common etiology, and the $\mathrm{C} 1-2$ joint is the most common site of VA occlusion [8].

The upper cervical spine is the most frequent affected site in patients with RA, causing severe neck pain, myelopathy, and sudden death in some cases [9]. AAS-induced fatal thromboembolic events in RA pa- 
tients were first described in 1968 [6]. Vertebrobasilar steno-occlusive disease in RA is known to result from various conditions of the spine. First, AAS can induce increased distortion and fragility of the VA as it runs around the transverse foramen of $\mathrm{C} 1-2$, particularly because of the VA passing oblique to the foramen of C1 [5]. Second, displacement of the odontoid process into the foramen magnum can induce constriction and stenosis of the VA between the odontoid process and the foramen magnum [10]. Third, destruction at the atlantoaxial joint can cause VA compression and narrowing [5]. In addition to those predisposing conditions, rotatory force to the neck can worsen the degree of VA steno-occlusive disease, which causes BHS in RA. Regarding the underlying etiology of the BHS in our case, we considered all of those that could be involved, and BHS was induced.

Previous investigators have demonstrated that some vertebrobasilar steno-occlusive diseases in RA are due to AAS and related vascular pathology, which are worsened by head rotation as described above. The mechanism of the vertebrobasilar infarction in our case is of interest. The underlying pathology in BHS patients is typically positional stenosis or occlusion of the VA, which induces temporary hemodynamic cerebral ischemia. In contrast to that, one previous investigator showed cases of embolic ischemia caused by artery-to-artery embolism after intimal injury [11]. We presumed that the mechanism of the infarction in our case was embolic ischemia due to intimal injury, rather than hemodynamic ischemia due to artery occlusion, because of the collateral flow from the contralateral VA demonstrated on DSA. Moreover, the VA is attached by fibrous tissue to the transverse foramen of $\mathrm{C}-2$; repeated head rotation can cause a severe stretch, inducing an intimal injury of the artery followed by cerebral embolism.

Although an arterial dissection should also have been considered, because of the etiology regarding the poor visualization of the right VA, our case did not show the typical findings for arterial dissection, such as visualization of subintimal hematomas or flaps on MRA and vessel wall images. We also did not find any luminal irregularity due to arterial dissection; hence we presumed that the repeated poor visualization of the right VA in the present case was caused by focal vasospasm due to the intimal injuries with mechanical compression rather than arterial dissection.

Regarding the mechanisms of the left PICA territory infarctions, we considered that the thrombus formed within the injured right VA embolized the left PICA through the connection between the left anterior inferior cerebellar artery (AICA) and the PICA, which is a well known anastomosis, the so-called AICA-PICA variant, in which AICA arises from the basilar artery and, in addition to its usual course and branching, nourishes the territory of the PICA as well [12].

Various kinds of etiology are known to induce vertebrobasilar insufficiency on MRI, such as poor visualization of the VA and vertebrobasilar infarctions. The common diseases showing such findings are intracranial atherosclerosis, dissection, and embolism from the extracranial region. In patients with RA, however, we should remember that findings are caused by embolic ischemia after intimal injury of the VA caused by neck rotation, such as BHS due to AAS and related vascular pathology. We should take BHS into consideration as a differential diagnosis in patients with vertebrobasilar insufficiency, especially in RA patients with repeated poor visualization of the VA.

Neurovascular imaging with head rotation is required for a correct diagnosis of BHS, but for less invasive vascular imaging, recent reports have demonstrated that doppler ultrasonography allows for useful measurements in the diagnosis of BHS [13]. Although its diagnostic ability may be limited, we can consider doppler ultrasonography with and without cervical rotation a diagnostic method for screening of BHS. In the present case, the diagnosis of BHS was delayed due to the lack of a useful diagnostic method such as doppler ultrasonography; therefore we should consider using that for screening of BHS in RA patients.

\section{Conclusion}

We reported a case of vertebrobasilar infarction due to BHS in a patient with RA. It is important for an accurate diagnosis of BHS to have neuroimaging with cervical rotation, especially for RA patients with repeated poor visualization of the VA. In our case, the infarction may have been caused by embolism due to intimal injury of the VA. 


\section{Conflict of Interest}

The authors declare that there is no conflict of interest.

\section{References}

1. Hernesniemi J \& Goehre F (2014): Rotational vertebral artery compression syndrome: bow hunter's stroke. World Neurosurg 82(5): 595-596

2. Lu DC, Zador Z, Mummaneni PV \& Lawton MT (2010): Rotational vertebral artery occlusion-series of 9 cases. Neurosurgery 67(4): 1066-1072

3. Weissman BN, Aliabadi P, Weinfeld MS, Thomas WH \& Sosman JL (1982): Prognostic features of atlantoaxial subluxation in rheumatoid arthritis patients. Radiology 144(4): 745-751

4. Fujiwara H, Kaito T, Makino T \& Yonenobu K (2012): Positional occlusion of the vertebral artery in a case of rheumatoid atlantoaxial subluxation presenting with multiple cerebral and cerebellar infarction. Mod Rheumatol 22(4): 605-609

5. Gaikwad SB, Suri A, Garg A, Kanodia A, Gupta V \& Mishralf NK (2004): Positional occlusion/stasis of vertebral arteries in a case of cervical rheumatoid arthritis presenting with multiple posterior circulation infarcts: a case report with angiographic demonstration. Spine (Phila Pa 1976) 29(15): E321-E325

6. Webb FW, Hickman JA \& Brew DS (1968): Death from vertebral artery thrombosis in rheumatoid arthritis. Br Med J 2(5604): 537
7. Takeshima Y, Matsuda R, Hironaka Y, Motoyama Y \& Nakase H (2015): Rheumatoid arthritis-induced lateral atlantoaxial subluxation with multiple vertebrobasilar infarctions. Spine (Phila Pa 1976) 40(3): E186-E189

8. Rastogi V, Rawls A, Moore O et al (2015): Rare etiology of bow hunter's syndrome and systematic review of literature. J Vasc Interv Neurol 8(3): 7-16

9. Neo M (2008): Treatment of upper cervical spine involvement in rheumatoid arthritis patients. Mod Rheumatol 18(4): 327-335

10. Jones MW \& Kaufmann JC (1976): Vertebrobasilar artery insufficiency in rheumatoid atlantoaxial subluxation. J Neurol Neurosurg Psychiatry 39(2): 122-128

11. Kageyama H, Yoshimura S, Iida T et al (2016): Juvenile cerebral infarction caused by bow hunter's syndrome during sport: two case reports. Neurol Med Chir (Tokyo) 56(9): 580-583

12. Baskaya MK, Coscarella E, Jea A \& Morcos JJ (2006): Aneurysm of the anterior inferior cerebellar artery-posterior inferior cerebellar artery variant: case report with anatomical description in the cadaver. Neurosurgery 58(2): E388; discussion E388

13. Dohzono S, Sasaoka R, Takamatsu K \& Nakamura H (2020): Bow hunter's syndrome after cervical laminoplasty in a patient with rheumatoid arthritis with bony ankylosis in the cervical spine: a case report. Mod Rheumatol Case Rep 4(1): 11-15

J UOEH $43(3): 349-353(2021)$ 